\title{
A little can be enough. Native fish from the Western Mediterranean Sea can act as a control agent for the invasive alga Caulerpa cylindracea
}

Jorge Santamaría ${ }^{1}$, Fiona Tomas ${ }^{2,3}$, Enric Ballesteros ${ }^{4}$, Juan Manuel Ruiz ${ }^{5}$, Jorge Terrados ${ }^{3}$, Emma Cebrian $^{1}$

${ }^{1}$ GRMAR Institute of Aquatic Ecology, University of Girona, Girona, Catalonia, Spain

${ }^{2}$ Department of Fisheries and Wildlife, Oregon State University, Corvallis, Oregon, United States of America

${ }^{3}$ Department of Ecology and Marine Resources - Instituto Mediterráneo de Estudios Avanzados (IMEDEA), Universitat de Les Illes Balears (UIB) - Consejo Superior de Investigaciones Científicas (CSIC), Esporles, Balearic Islands, Spain

${ }^{4}$ Centro d’Estudis Avançats de Blanes, Consejo Superior de Investigaciones Científicas (CEAB-CSIC), Blanes, Catalonia, Spain

${ }^{5}$ Seagrass Ecology Group - Centro Oceanográfico de Murcia, Instituto Español de Oceanografía (IEO), San Pedro del Pinatar, Murcia, Spain

Corresponding Author:

Jorge Santamaría ${ }^{1}$

Email address: jorge.santamaria@udg.edu 


\title{
A little can be enough. Native fish from the Western Mediterranean Sea can act as a control agent for the invasive alga Caulerpa cylindracea
}

\author{
Jorge Santamaría ${ }^{1}$, Fiona Tomas ${ }^{2,3}$, Enric Ballesteros ${ }^{4}$, Juan Manuel Ruiz ${ }^{5}$, Jorge Terrados ${ }^{3}$, \\ Emma Cebrian $^{1}$ \\ ${ }^{1}$ GRMAR Institute of Aquatic Ecology, University of Girona, Girona, Catalonia, Spain \\ ${ }^{2}$ Department of Fisheries and Wildlife, Oregon State University, Corvallis, Oregon, United \\ States of America \\ ${ }^{3}$ Department of Ecology and Marine Resources - Instituto Mediterráneo de Estudios \\ Avanzados (IMEDEA), Universitat de Les Illes Balears (UIB) - Consejo Superior de \\ Investigaciones Científicas (CSIC), Esporles, Balearic Islands, Spain \\ ${ }^{4}$ Centro d'Estudis Avançats de Blanes, Consejo Superior de Investigaciones Científicas \\ (CEAB-CSIC), Blanes, Catalonia, Spain \\ ${ }^{5}$ Seagrass Ecology Group - Centro Oceanográfico de Murcia, Instituto Español de \\ Oceanografía (IEO), San Pedro del Pinatar, Murcia, Spain
}

1 In the Mediterranean Sea, different organisms can feed on invasive algae species, yet, 2 how these species provide biotic resistance against algal invasions remains unclear. In 3 this study, we analyzed fish stomach contents to determine which fish species feed on

4 Caulerpa cylindracea and we performed an exclusion experiment to experimentally test 5 how this grazing activity may limit invasive algae abundance and spread. Our results 6 show that several fish species, many of them not considered strictly herbivores, feed on 7 the invasive alga; however, the Ivlev's Index suggests that its consumption was 8 accidental except for Sarpa salpa. Additionally, the exclusion experiment demonstrated 9 that fish species can limit C. cylindracea coverage at $10 \mathrm{~m}$ but not at $30 \mathrm{~m}$ deep; which is 10 likely linked to the higher abundance and activity of these fish species at depths above $1125 \mathrm{~m}$. These results are in agreement with the current distribution of C. cylindracea, 12 which is much more abundant at depths from 25 to $50 \mathrm{~m}$. In this study we show that fish 13 herbivory is a form of biotic resistance against C. cylindracea at shallow depths, not 14 being able to completely remove it, but controlling its abundance. 
(C) 2018 Santamaría et al. This is an open access article distributed under the terms of the Creative Commons Attribution License:

http://creativecommons.org/licenses/by/4.0/ (the "License"), which permits unrestricted use, distribution, reproduction and adaptation in any medium and for any purpose provided that it is properly attributed. For attribution, the original author(s), title, publication source (PeerJ Preprints) and either DOI or URL of the article must be cited. Notwithstanding the ProQuest Terms and Conditions, you may use this content in accordance with the terms of the License. 